\title{
Distances, depth structure, and kinematics of the Magellanic Clouds from disentangling spectra of eclipsing binaries and Cepheids
}

\author{
P. Hadrava, ${ }^{1}$ S. Štefl, ${ }^{2}$ R. Klement,${ }^{3}$ and C. Martayan ${ }^{2}$ \\ ${ }^{1}$ Astronomical Institute, Academy of Sciences, Boční II 1401, CZ 14131 Praha 4, \\ Czech Republic \\ email: had@sunstel.asu.cas.cz \\ ${ }^{2}$ European Organisation for Astronomical Research in the Southern Hemisphere, \\ Casilla 19001, Santiago 19, Chile \\ email: sstefl@eso.org, cmartaya@eso.org \\ ${ }^{3}$ Department of Theoretical Physics and Astrophysics, Masaryk University, CZ 61137 Brno, \\ Czech Republic \\ email: robertklement@gmail.com
}

\begin{abstract}
Spectroscopy and photometry of eclipsing binaries and Cepheids (using the BaadeWesselink method) yield primary distance markers applicable to the Local Group of galaxies. The technique of disentangling spectra has proved helpful in resolving stellar physical parameters. Our method also allows us to disentangle interstellar absorption lines and, thus, to complement the radial-velocity structure of the interstellar medium with an (otherwise unattainable) upper limit to its distance. The mapping of the spatial and kinematic structure of the stellar and interstellar components of the Magellanic Clouds and Stream enables constraining their tidal evolution and, thus, of the mass of the Galaxy.
\end{abstract}

Keywords. binaries: eclipsing, Cepheids, ISM: kinematics and dynamics, Magellanic Clouds

\section{Introduction}

Observations of variable stars in the Local Group of galaxies have provided a gateway to the cosmological distance scale ever since the discovery of the Cepheid period-luminosity (PL) relation by Leavitt (1908). The PL relation still requires further sophistication, both observationally and theoretically, for several reasons: (i) an increase in the precision of distance determination can improve calibrations of secondary methods applicable to the more distant Universe; (ii) a better knowledge of the physical properties of individual stars in the Magellanic Clouds and other nearby galaxies places constraints on the theory of stellar structure and evolution for different elemental abundances as well as on the chemical evolution of the Universe in general; and (iii) precise mapping of the depth structure and kinematics of the Magellanic system enables us to trace its motion with respect to the Milky Way (MW) and to investigate the tidal interactions between the Large and Small Magellanic Clouds (LMC, SMC) on the one hand, and with the MW on the other, which offers clues to the mass of the MW and its distribution, including of the dark matter content (Růžička et al. 2007).

Complete knowledge of the distribution of the SMC/LMC's stellar component as well as of the interstellar matter (ISM) in phase-space is desirable. While information about the angular direction $\{\alpha, \delta\}$, distance, proper motion and radial velocity (RV) is, in principle, available for the stars, the density of the ISM can only be mapped using data cubes, $\{\alpha, \delta, \mathrm{RV}\}$, based on observations at radio frequencies. Our method of Fourier 
disentangling of stellar spectra (Hadrava 1995, 1997, 2004, 2009, 2012), which facilitates the determination of the physical properties of both binary components and Cepheids and, consequently, also of their distances, allows us to separate the spectral lines of the ISM and to distinguish them from stellar photospheric lines. For ISM absorption lines, the stellar distance is thus an upper limit to the distance of the ISM. Here we give a brief account of this application of spectral disentangling.

\section{Binaries and Cepheids as primary distance markers}

The Cepheid PL relation must be calibrated to be usable for distance measurements. However, its calibration can also be based on the first principles of physics in different incarnations of the Baade (1926)/Wesselink (1946) method. This method is used to derive - from spectroscopically observed line-profile variations (LPVs) - the absolute changes in the radii of Cepheids, i.e., it provides a standard ruler useful for purely geometric measurements of distances from interferometric observations (cf. Kervella et al. 2004), or, in combination with the determination of the (variable) atmospheric temperature, a standard candle for photometric distance measurements.

The common practice of determining changes in Cepheid radii from measurements of RVs using some standard method (e.g., based on moments) and of calibrating them using a so-called 'projection factor' violates the requirements for a primary method. Our method of generalized disentangling, which can also include LPVs, enables us to directly obtain the time derivatives of the radii (cf. Hadrava et al. 2009). It also yields the instantaneous strengths of spectral lines originating from different ionic excitation levels, which indicate temperature variations. Our results for $\delta$ Cep also reveal differences between RV curves of these excitation levels, which show diferential motions in the pulsating atmosphere and the need for a more complex model.

Similarly, RV curves of binaries provide information about their absolute orbital dimensions, i.e., a standard ruler for purely geometric determination of distances to interferometrically resolved binaries. Standard spectral disentangling, i.e., a simultaneous separation of component spectra and determination of binary orbital parameters, is a convenient and precise tool for measurement and solution of RV curves. It was used, e.g., to resolve the Pleiades paradox by Zwahlen et al. (2004). The separated spectra can then be used for determination of the physical properties of the binary components.

Another option to use spectroscopic binaries for distance measurements is offered by the photometric method. It is applicable to eclipsing binaries, for which the orbital inclination and the components' radii in units of the obital size can be determined from their light curves. To get a standard candle, the luminosities of the components must also be derived from their effective temperatures, $T_{\text {eff }}$. These can be obtained from multicolour photometry or from the spectra of the individual components, which are separated in the process of disentangling. It is well-known that the disentangled spectra can be determined up to a multiplicative factor corresponding to the ratio of the component continua. This ratio can be derived from the light curves. Because the disentangled spectra are usually compared with synthetic spectra to obtain $T_{\text {eff }}$ and other atmospheric parameters (usually by employing least-squares minimization, which is also the basis of the disentangling approach), an alternative method (applicable even to non-eclipsing binaries) can be used to restrict the disentangled spectra based on templates interpolated from a grid of models. One can then include their parameters together with the multiplicative factors between the optimized parameters. (There is also a simpler way to disentangle the spectra using the discrete set of synthetic templates, by finding the minimum of the residual noise at these grid points.) Note that the gravitational acceleration, $\log g$, is 

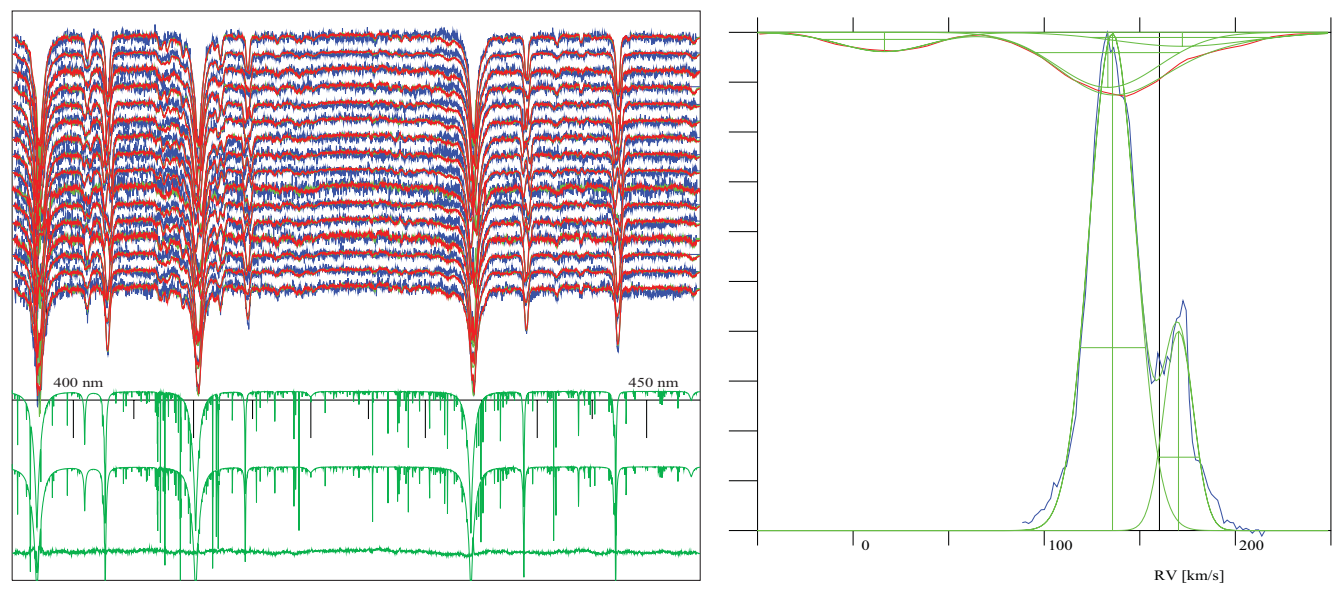

Figure 1. (left) 16 VLT/FLAMES spectra of the eclipsing binary SMC-SC5 38089 disentangled using model templates for the component stars and a free interstellar component. (right) Detail of the disentangled CaII interstellar absorption compared with $\mathrm{HI}$ emission at $21 \mathrm{~cm}$.

better determined from the orbital solution and the radii obtained from the light curves. Generally, orbital and other parameters are constrained by both the spectroscopy and photometry. It is thus optimal to generalize the disentangling to a simultaneous solution of all available data by fitting them with theoretical models as a function of a set of properly chosen free parameters, which may also include the distance (cf. Wilson 2008). Our new code, BAZANT (under development), which merges our previous code, FOTEL (used for analysis of light and RV curves, etc.), with KoREL for Fourier disentangling, is a first step towards such a complex tool for interpretation of observational data.

Unfortunately, the Cepheids' disks and the individual components of spectroscopic binaries can only be resolved by present-day interferometric setups for targets in the MW. Hence, the purely geometric method does not reach even the other galaxies in the Local Group. The photometric methods can thus only be applied to this distance range. These methods suffer from an additional complication caused by interstellar extinction. This is usually estimated from multicolour photometry based on reddening. The disentangling of interstellar lines yields an additional tool for its estimation.

\section{Disentangling of eclipsing binaries in the Small Magellanic Cloud}

We have applied this procedure to several early-type binaries in the SMC (Klement 2012). The photometry was taken from the OGLE II survey (Udalski et al. 1998), the spectroscopy was obtained with the FLAMES/GIRAFFE multiobject spectrograph at the Very Large Telescope (the spectra were obtained and analyzed by North et al. 2010). We have reduced the spectra from the ESO archive with the GIRAFFE pipeline, version 2.8.9. The orbital parameters of selected systems were obtained using the BAzANT code, applied to a set of narrow spectral regions with high resolution around strong helium and hydrogen lines. The hydrogen lines are usually ignored by other authors because of frequent contamination by interstellar emission. However, this is not a serious obstacle for our approach using variable line-strength factors (cf. Hadrava 1997), which enables us to disentangle the interstellar contribution to line profiles as a third component with constant RVs (the line strengths of this component differ irregularly between exposures because the ratio of signal from the point-like star and the diffuse interstellar background depends on variations in the seeing and irregularities in the pointing). Another outdated, 
bad habit persisting from the classical methods of RV measurements is to avoid exposures taken near the conjunctions of the binary system. The technique of disentangling enables us to measure RVs even for highly blended lines in these phases, which are valuable for determination of periods and other orbital parameters. Moreover, each exposure helps to improve the signal-to-noise ratio of the disentangled spectra and hence the accuracy of the entire solution. For exposures taken at eclipses of fast rotators, the disentangling should make use of LPVs because of the effects of rotation (cf. Hadrava 2009). However, for the commonly occurring partial eclipses of polar regions of slow rotators, changes in line-profile shapes are negligible and it is usually sufficient to take into account changes in line strengths during eclipses. To find the effective temperatures of the components and thus also their luminosities (for distance determination), we disentangled the entire available spectral regions with various templates based on synthetic spectra (cf. Fig. 1, left). The orbital parameters were fixed based on the previous solution of narrow spectral regions, while the rotational broadening and systemic velocities converged.

Disentangling of interstellar lines enabled us to separate a strong Fraunhofer CaII $\mathrm{H}$ absorption line in the wing of the $\mathrm{H} \varepsilon$ Balmer line. It is partially blended with inter/circumstellar $\mathrm{H} \varepsilon$ emission in some binaries. However, the latter can be distinguished and separated by considering the variability in strength. In Fig. 1 (right) we show an example of the interstellar CaII absorption towards the star SMC-SC5 38089. It can be approximated by a superposition of three Gaussian profiles, one of Galactic origin at RV $\simeq 16.4 \mathrm{~km} \mathrm{~s}^{-1}$ and two with RVs of 133.3 and $172.3 \mathrm{~km} \mathrm{~s}^{-1}$. The latter correspond to the RV distribution of the HI emission observed at RVs of 135.7 and $170.3 \mathrm{~km} \mathrm{~s}^{-1}$. These two peaks thus obviously originate along the line of sight to the binary, which has RV = $160.3 \mathrm{~km} \mathrm{~s}^{-1}$. The larger widths of the absorption components correspond to the lower resolution ( $R=6400$, i.e., approximately $50 \mathrm{~km} \mathrm{~s}^{-1}$ ) of the optical spectrograph.

\section{Acknowledgements}

This work uses ESO and OGLE archive data. It has been supported by projects RVO:679 85815, GAČR 202/09/0772, MUNI/A/0968/2009, and the ESO students programme.

\section{References}

Baade, W. 1926, Astron. Nachr., 228, 359

Hadrava, P. 1995, A\&AS, 114, 393

Hadrava, P. 1997, A\&SAS, 122, 581

Hadrava, P. 2004, Publ. Astron. Inst. Acad. Sci. Czech Rep., 92, 15

Hadrava, P. 2009, Disentangling of spectra: theory and practice, arXiv:0909.0172

Hadrava, P. 2012, in: From Interacting Binaries to Exoplanets: Essential Modeling Tools (Richards, M. T., \& Hubeny, I., eds.), Proc. IAU Symp., 282, 351

Hadrava, P., Šlechta, M., \& Škoda, P. 2009, A $\& A$ A, 507, 397

Kervella, P, Fouqué, P., et al. 2004 ApJ, 604, L113

Klement, R. 2012, Diploma thesis, Masaryk Univ. Brno, Czech Rep. (https://is.muni.cz/th/269086/prif_m/diplomka_lecldsnr.pdf)

Leavitt, H. S. 1908, Ann. Harvard Coll. Obs., 60, 87

North, P., Gauderon, R., Barblan, F., \& Royer, F. 2011, A\&A, 520, A74

Růžička, A., Palouš, J., \& Theis, C. 2007, A\&A, 461, 155

Udalski, A., Soszynski, I., Szymanski, M., et al. 1998, Acta Astron., 48, 563

Wesselink, A. J. 1946, Bull. Astron. Inst. Neth., 10, 91

Wilson, R. E. 2008, ApJ, 672, 575

Zwahlen, N., North, P., Debernardi, Y., et al. 2004, A\&A, 425, L45 